\title{
Guest editorial: Introducing progress in hematology in this issue
}

\author{
Tomoki Naoe
}

Received: 9 July 2012/Revised: 17 July 2012 / Accepted: 17 July 2012/Published online: 6 August 2012

(C) The Japanese Society of Hematology 2012

This issue of IJH contains five serial review articles on acute myeloid leukemia (AML). AML is a molecularly heterogeneous neoplasm, and the understanding of its molecular pathogenesis continues to grow. The development of chemotherapy and hematopoietic stem cell transplantation (HSCT) have increased 5-year survival rate to $40 \%$ in AML patients younger than 60-65 years, whereas outcome in elderly AML patients remains dismal.

In the first review, David Grimwade summarizes the molecular heterogeneity of AML and clinical significance of each molecular abnormality. In his work with the UK Medical Research Council (MRC), he has published important articles on prognostic factors in AML and characterization of the mechanisms underlying leukemiaassociated chromosomal translocations. A consensus had emerged that leukemogenesis requires two classes of mutations in AML (WHO classification 2008). The first of these is abnormalities in transcriptional factors that impair hematopoietic differentiation. Rearrangement of genes such as RUNX1, RARA, or MLL belongs to this class of mutations as well as point mutation of CEBPA (these are known as class II mutations). The second class of mutations consists of FLT3, KIT, or NRAS mutations (class I mutations), which are associated with cellular proliferation and/or survival. The discovery of the NPMI mutation in 2007 , however, suggested that the story is not so simple. From 2009, genome sequence analysis revealed novel gene mutations such as TET2, IDH1/2, and DNMT3A especially in AML showing normal karyotype. These gene products

\section{T. Naoe $(\square)$}

Department of Hematology and Oncology,

Nagoya University Graduate School of Medicine,

Nagoya 466-8550, Japan

e-mail: tnaoe@med.nagoya-u.ac.jp are associated with epigenetic modifications, including DNA methylation, and their biological significance in leukemogenesis is under investigation.

In the second perspective, Martin Tallman, chairman of the Leukemia Committee for the Eastern Cooperative Oncology Group (ECOG), reviews induction chemotherapy for AML. Although the fundamental drug combination for remission-induction, DNR or IDA, plus Ara-C, has not changed for more than 30 years, there is still no definitive protocol for dose and schedule. Furthermore, many trials are under way seeking to improve $\mathrm{CR}$ rate and survival; these experimental approaches include intensification of anthracycline, high-dose of Ara-C, addition of a third drug, priming usage of G-CSF, and combination with molecular targeted drug.

Third, co-chairman of the Japan Adult Leukemia Study Group (JALSG) Shuichi Miyawaki was invited to overview Japanese studies on AML. The JALSG has just celebrated its 25th anniversary with the holding of an international symposium at Tokyo Forum on June 23-24, 2012, which this review article commemorates. JALSG has completed six studies of AML to date. As Ryuzo Ohno previously reviewed the first four JALSG studies from 1987 to 1997 (Int J Hematol. 72:272, 2000), Miyawaki focused primarily on the two studies after 1997.

Working with Masamitsu Yanada, I co-authored the fourth review, focusing on elderly AML, which is becoming a more important issue in many countries. In the JALSG data, the median age of newly diagnosed patients with AML and high-risk MDS is around 65 years. Accordingly, at least half of AML patients are not included in clinical studies using intensive chemotherapy and HSCT. Since conventional therapy has limitations in improving outcomes in elderly AML, new therapeutic approaches are urgently needed. 
Finally, Bruno Medeiros, a speaker at the recent JALSG symposium, was invited to describe novel agents in AML. Despite the use of intensified chemotherapy and/or HSCT, the overall 5-year survival of aged patients with AML remains less than $25 \%$, which is remarkably low compared with other hematopoietic malignancies. One of the reasons is lack of new breakthrough drugs in AML. However, the accumulated understanding of the molecular biology of
AML has identified numerous therapeutic targets, and a growing number of promising agents are under clinical development.

I hope that these five excellent articles would help readers to deepen their understanding of the state-of-thescience in AML, and provide insights into the future of the field. 